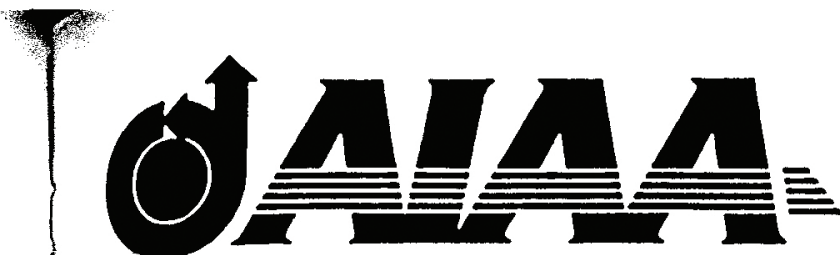

\title{
AIAA 95-0104 \\ The Direct Measurement of Circulation in Free Surface Vortices
}

R. H. Smith, Jr., W. W. Durgin, and

H. Johari

Mechanical Engineering Department

Worcester Polytechnic Institute

Worcester, MA

\section{3rd Aerospace Sciences Meeting and Exhibit January 9-12, 1995 / Reno, NV}




\title{
The Direct Measurement of Circulation in Free Surface Vortices
}

\author{
R. H. Smith, Jr., W. W. Durgin ${ }^{\dagger}$, H. Johari ${ }^{\ddagger}$ \\ Mechanical Engineering Department \\ Worcester Polytechnic Institute \\ Worcester, MA 01609
}

\begin{abstract}
Ultrasonic techniques have been used to directly and non-intrusively measure the circulation of free surface vortices. All experiments were performed in a vertical cylindrical tank with a central drain and a tangential inlet. The circulation was measured on a closed triangular path by measuring the difference in upstream and downstream transit-times. Circulation was measured as a function of the Reynolds and Froude numbers and was found to increase as the Reynolds and Froude numbers increased. The circulation was also found to be proportional to the square of the ratio of the drain diameter to cylinder diameter while the ratio of fluid depth to cylinder diameter was held constant. Minimum surface elevations were measured at various conditions and attempts were made to correlate them with measured circulation.
\end{abstract}

\section{Nomenclature}

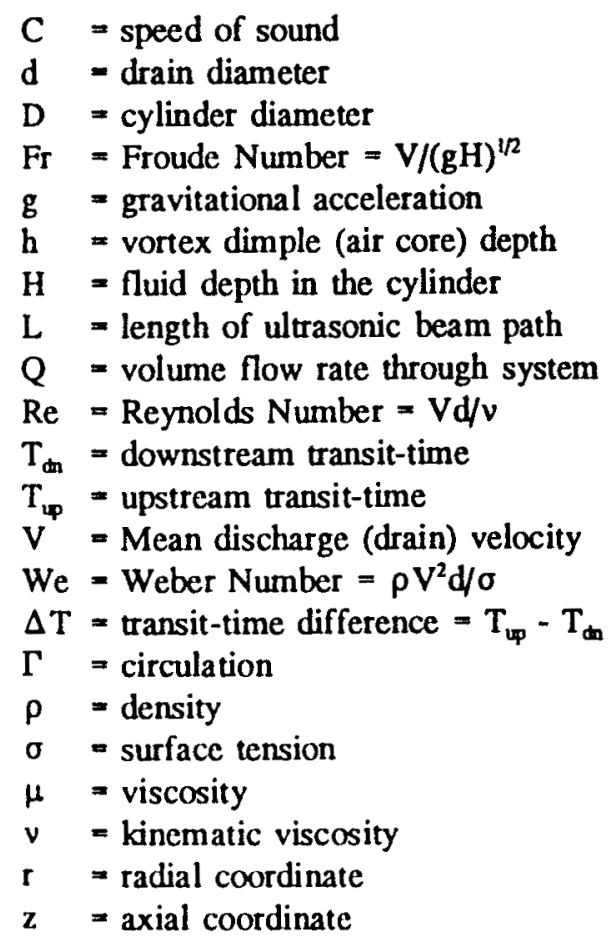

*Graduate Student, Member AlAA

'Professor, Member AIAA

'Assistant Professor, Member AIAA

Copyright $\odot 1994$ by R. H. Smith, Jr., W. W. Durgin, and $\mathrm{H}$. Johari, Worcester Polytechnic Institute. Published by the American Institute of Aeronautics and Astronautics, Inc. with permission.

\section{Background}

The rotating flow that is formed when a container is drained is an example of a free surface vortex. The size and strength of such vortices is an important consideration in many engineering applications such as fuel and liquid transfers at various gravity levels, the draining of storage tanks or fuel draw down in rockets, at the inlets to pumps and piping systems, and in hydraulic structures and fluid machinery. ${ }^{1-6}$

The free surface vortex is a complex, threedimensional, viscous flow field with unsteady axial, radial, and tangential velocity components that vary spatially. A relatively stable vortex can be formed in cylindrical tanks and many studies have been preformed to characterize the flow field. ${ }^{8-10}$ The flow in the outlet may possess considerable axial velocity, stretching and amplifying the vortex filaments. The radial velocity component has been found to vary with both radius, $r$, and axial position, $z^{7,8}$ The tangential velocity component of the flow has been found to be inversely proportional to radius and independent of depth except for a small region near the bottom of the tank where the boundary layer affects the flow. ${ }^{7-9}$

Measurement of the three velocity components in free surface vortices is difficult because the vortex typically tends to move within the container. Measurements of the velocity components have been undertaken through various methods. ${ }^{7.9}$ A serious problem in taking measurements in vortical flows is that physical probes tend to affect the flow. Qualitative classification of vortex strength using visual methods was pursed by Durgin and Hecker. ${ }^{10}$ In the present effort, ultrasonic techniques were used to directly, and non-intrusively measure the circulation of free surface vortices. 


\section{Dimensional Arguments}

Figure 1 shows a general representation of a free surface vortex and the cylindrical tank. The following parameters were determined to be important to the problem under study: gravitational acceleration $\mathrm{g}$, drain diameter $d$, cylinder diameter $D$, dimple (air core) depth $h$, fluid depth $H$, circulation $\Gamma$, fluid density $\rho$, fluid viscosity $\mu$, mean drain velocity $V$, and fluid surface tension $\sigma$. Dimensional reasoning provides the following functional relationships for the circulation:

$$
\frac{\Gamma}{V d}=F_{1}\left(R e, F r, W e, \frac{d}{D}, \frac{H}{D}\right)
$$

and for the air core depth:

$$
\frac{h}{H}=F_{2}\left(\operatorname{Re}, F r, W e, \frac{d}{D}, \frac{H}{D}\right)
$$

where:

$$
\begin{gathered}
\operatorname{Re}=\frac{\mathrm{Vd}}{v} \quad \mathrm{Fr}=\frac{\mathrm{V}}{\sqrt{\mathrm{gH}}} \\
\mathrm{We}=\frac{\rho \mathrm{V}^{2} \mathrm{~d}}{\sigma}
\end{gathered}
$$

Daggett \& Keulegan ${ }^{8}$ found the same parameters for similitude although their arrangement is slightly different. For the present experiments under consideration, the cylinder diameter $D$, the free surface depth $\mathrm{H}$, and of course gravity $\mathrm{g}$ were held constant. The fluid properties $\rho, \mu$, and $\sigma$ were assumed to remain constant since the experiments were preformed in an area with nearly constant temperature. No precautions were taken to control these parameters. The drain diameter $\mathrm{d}$ and the volume flow rate $(\mathrm{Q}=$ $\left.\left(\pi V d^{2}\right) / 4\right)$ were varied independently. The volume flow rate and the drain diameter are used to calculate the drain velocity which is used to calculate the Reynolds, Froude, and Weber numbers. The drain velocity is also used to non-dimensionalize the circulation. Previous measurements ${ }^{8}$ have indicated that Weber number effects on the discharge coefficient and circulation are small.

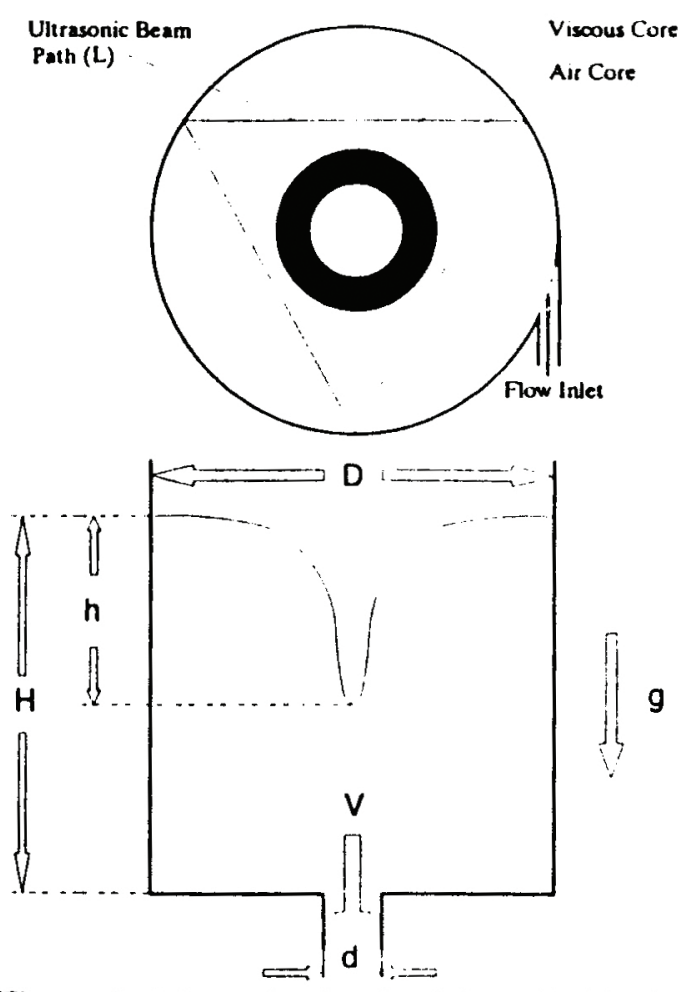

Figure 1. Schematic sketch of the cylindrical tank and relevant parameters

\section{Experimental Apparatus}

The experiment was designed to establish a stable free surface vortex. The experimental apparatus consisted of a cylindrical tank, a pump, a needle valve, a 1/2" offset flow cell", two Panametrics PT 868 Ultrasonic flowmeters, four $1 \mathrm{MHz}$ ultrasonic transducers and a cathatometer. Distilled, deionized water was used as the working fluid. A block diagram of the apparatus is shown in Figure 2.

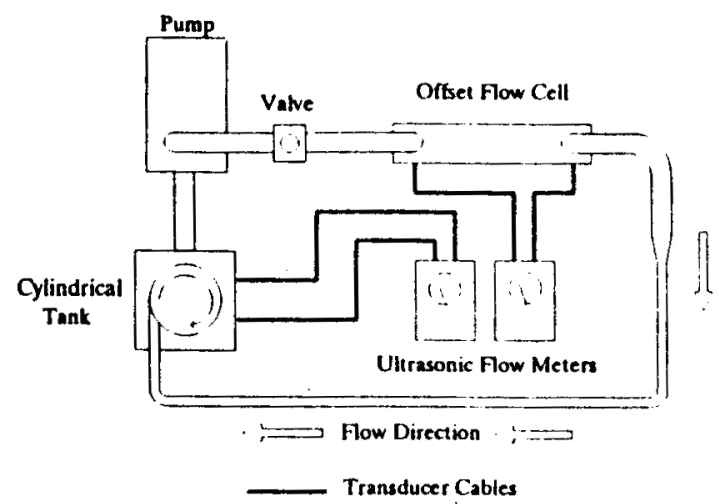

Figure 2. Experimental apparatus block diagram 
The water was drawn out of the central drain at the bottom of the cylindrical tank and pumped through an offset flow cell for volume flow rate measurement, and back into the tank through an inlet located on the side of the cylinder near the free surface. A constant mean fluid depth $(\mathrm{H}=24.4 \mathrm{~cm})$ was maintained in the tank A magnetic drive centrifugal pump was used to maintain a constant volume flow rate controlled by a needle valve; the valve regulates the size (air core depth, h) and strength of the vortex formed. The cylindrical geometry, constant mean fluid depth, and constant volume flow rate allowed stable vortices to be formed. The offset flow cell was used in conjunction with two $1 \mathrm{MHz}$ ultrasonic transducers and one of the ultrasonic flowmeters to measure and record the volummetric flow rate during the experiment. The offset flow cell was only used to measure $Q ;$ a different transducer arrangement was used with a second PT868 flowmeter to measure the circulation.

The cylindrical tank, shown in Figure 3, has an inner diameter of $15.2 \mathrm{~cm}$ and a depth of $28 \mathrm{~cm}$. The cylindrical tank consisted of an upper and lower transparent lucite cylinders, an aluminum top and bottom plate, and an aluminum ultrasonic transducer mounting block.

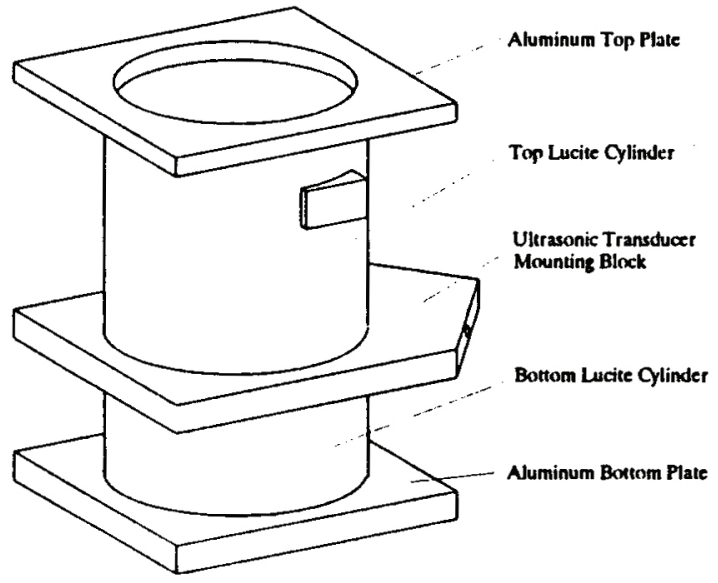

Figure 3. Cylindrical tank

The flow inlet was located approximately tangent to the inner surface of the upper lucite cylinder and nearly level with the free surface so as not to disturb the normal vortex flow field; the inlet had a diameter of $0.318 \mathrm{~cm}$. The flow outlet was located in the center of the aluminum bottom plate. The drain diameter could be varied from $0.318 \mathrm{~cm}$ to $1.588 \mathrm{~cm}$ in $0.318 \mathrm{~cm}$ increments. The outlet had a sharp lip and extended 5 drain diameters downstream. A schematic of the bottom plate is shown in Figure 4.
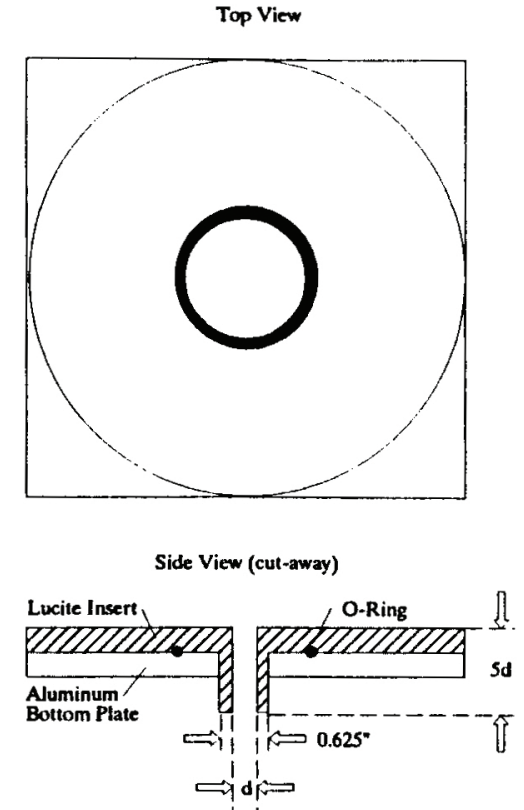

Figure 4. Schematic of the bottom plate and details of the outlet lip

Ultrasonic flowmeters work on the principle that the propagation speed of sound waves in a fluid changes when a component of the fluid velocity is parallel to the direction of the sound wave. ${ }^{12-15}$ A simple single path setup for an ultrasonic flowmeter is shown in Figure 5.

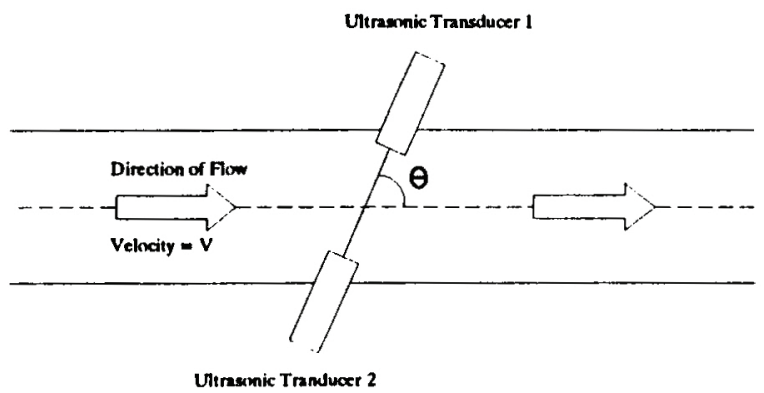

Figure 5. Simple ultrasonic flowmeter after Lowell ${ }^{13,15}$

Both ultrasonic transducers are capable of transmitting and receiving a sound pulse. The distance between the two transducers is the path length, $L$. The time it takes for an ultrasonic pulse to travel from one transducer to the other is the transit-time. The transittime measured against the flow direction for a constant $\mathrm{V}$ along the path, from transducer 1 to transducer 2 , is know as the upstream travel time ${ }^{13}, T_{\mathrm{Lp}}$ :

$$
\mathrm{T}_{\mathrm{up}}=\frac{\mathrm{L}}{\mathrm{C}-\mathrm{V} \cos \theta}
$$


The transit-time measured with the flow, from transducer 2 to transducer 1 , is known as the downstream travel time, $T_{d n}$ :

$$
T_{d n}=\frac{L}{C+V \cos \theta}
$$

The time difference, $\Delta T$, between $T_{u p}$ and $T_{d a}$ is proportional to the fluid velocity:

$$
\Delta \mathrm{T}=\mathrm{T}_{\text {up }}-\mathrm{T}_{\mathrm{dn}}=\frac{2 \mathrm{VL} \cos \theta}{\mathrm{C}^{2}-\mathrm{V}^{2} \cos ^{2} \theta}
$$

Since in water, $\mathrm{C} \gg \mathrm{V}$,

$$
\Delta \mathrm{T}=\frac{2 \mathrm{VL} \cos \theta}{\mathrm{C}^{2}}
$$

By knowing $L$ and measuring the transit-times without any flow, $C$ can be found very accurately. The flow velocity, V, can now be obtained by measuring the transit-time difference.

The ultrasonic transducer mounting block, shown in Figure 6, is used to create a closed triangular beam path over which the circulation can be measured by using the transit-time measurement technique.

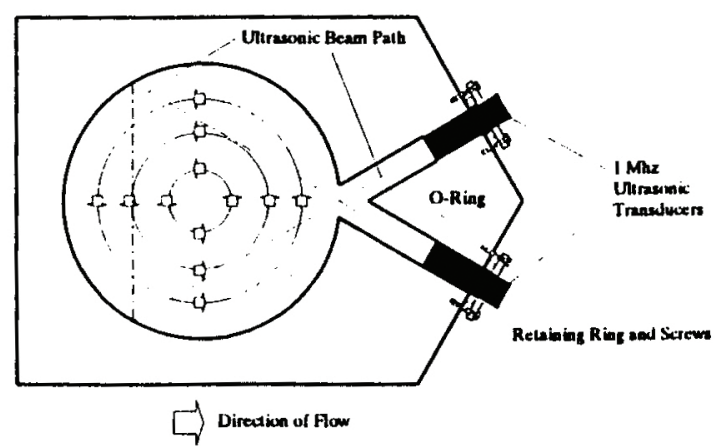

Figure 6. Schematic of ultrasonic mount

The time difference for the triangular path can be calculated by integrating equation 6 over the entire path; the tangential velocity as well as the local angle $\theta$ change as the sound pulse traverses the path. Equation 6 may be rewritten as

$$
\Delta \mathrm{T}=6 \int_{0}^{\mathrm{L} / 6} \frac{2 \mathrm{~V}_{\theta} \cos \theta}{\mathrm{C}^{2}-\mathrm{V}_{\theta}^{2} \cos ^{2} \theta} \mathrm{dl}
$$

Previous measurements ${ }^{8}$ have shown that the tangential velocity component of free surface vortices varies inversely with radius for $\mathrm{r}$ greater than $\mathrm{d} / 2$, except very near the bottom boundary layer. Taking the tangential velocity $V_{\theta}$ to be $\Gamma /(2 \pi r)$, equation 8 can be integrated to reveal

$$
\Gamma=0.605\left(C^{2} \Delta T\right)
$$

The contributions of the radial velocity component cancel around a closed circuit. $\Delta \mathrm{T}$ is measured by one of the PT868 flowmeter. For the strongest vortices, the largest $\Delta \mathrm{T}$ measured was approximately 95 nanoseconds. In still water, the transit times were equal: $T_{u p}=T_{d n}=230 \mu$-seconds. Accuracy of a single $\Delta \mathrm{T}$ measurement is $\pm 2 \%$.

The transit-time difference, $\Delta \mathrm{T}$, and volume flow rate, $Q$, were measured in 125 experimental runs: 31 at $\mathrm{d}=1.588 \mathrm{~cm}, 30$ at $\mathrm{d}=1.27 \mathrm{~cm}, 31$ at $\mathrm{d}=0.953 \mathrm{~cm}$, 18 at $\mathrm{d}=0.635 \mathrm{~cm}$, and 15 at $\mathrm{d}=0.318 \mathrm{~cm}$. The mean volume flow rate and the transit-time difference were measured and recorded every five seconds for five minutes. The sixty data points were then averaged to obtain values for the experimental run. The vortex dimple depth was measured with a cathatometer. The ratio $\mathrm{h} / \mathrm{H}$ was measured with an accuracy of $\pm 2 \%$ over most of the range; at very small and very large $\mathrm{h} / \mathrm{H}$ values, the air core depth was difficult to measure and the error is greater. The Reynolds and Froude numbers were calculated from equation (3) and their estimated uncertainty is $\pm 3 \%$. The circulation was calculated from equation (9) and was measured with an accuracy of $\pm 2 \%$. Details of the experimental setup can be found in Smith ${ }^{16}$.

\section{Results and Discussion}

Figures 7 and 8 show the results of the circulation measurements for the $1.588 \mathrm{~cm}$ and the $0.635 \mathrm{~cm}$ drain diameters, respectively; Figures 10 and 11 , show the results of the $\mathrm{h} / \mathrm{H}$ measurements for the $1.588 \mathrm{~cm}$ and the $0.635 \mathrm{~cm}$ drain diameters, respectively. These results are typical of the five drain diameters tested. The measured circulation and the ratio of air core depth to cylinder water depth, $h / H$, are plotted against the Reynolds and Froude numbers. The Reynolds and Froude numbers varied from 500 to 6500 and from 0.02 to 1.5 , respectively. The plotted data do not take into account possible variations of the Weber number.

Figures 7 (a) and 8 (a) show circulation, $\Gamma\left(\mathrm{Fr}^{2} / \mathrm{s}\right)$ plotted against Reynolds and Froude numbers. Figures 7 (b) and 8 (b) show the projection of the experimental data on the Reynolds- $\Gamma$ plane. Figures 7 (c) and 8 (c) show the projection of the data on the Froude- $\Gamma$ plane. From the graphs it can be seen that circulation increases essentially linearly with both Reynolds and Froude numbers. It is noteworthy that Froude and Reynolds numbers variations are due to the velocity while $d, H$, and $v$ are held constant. 

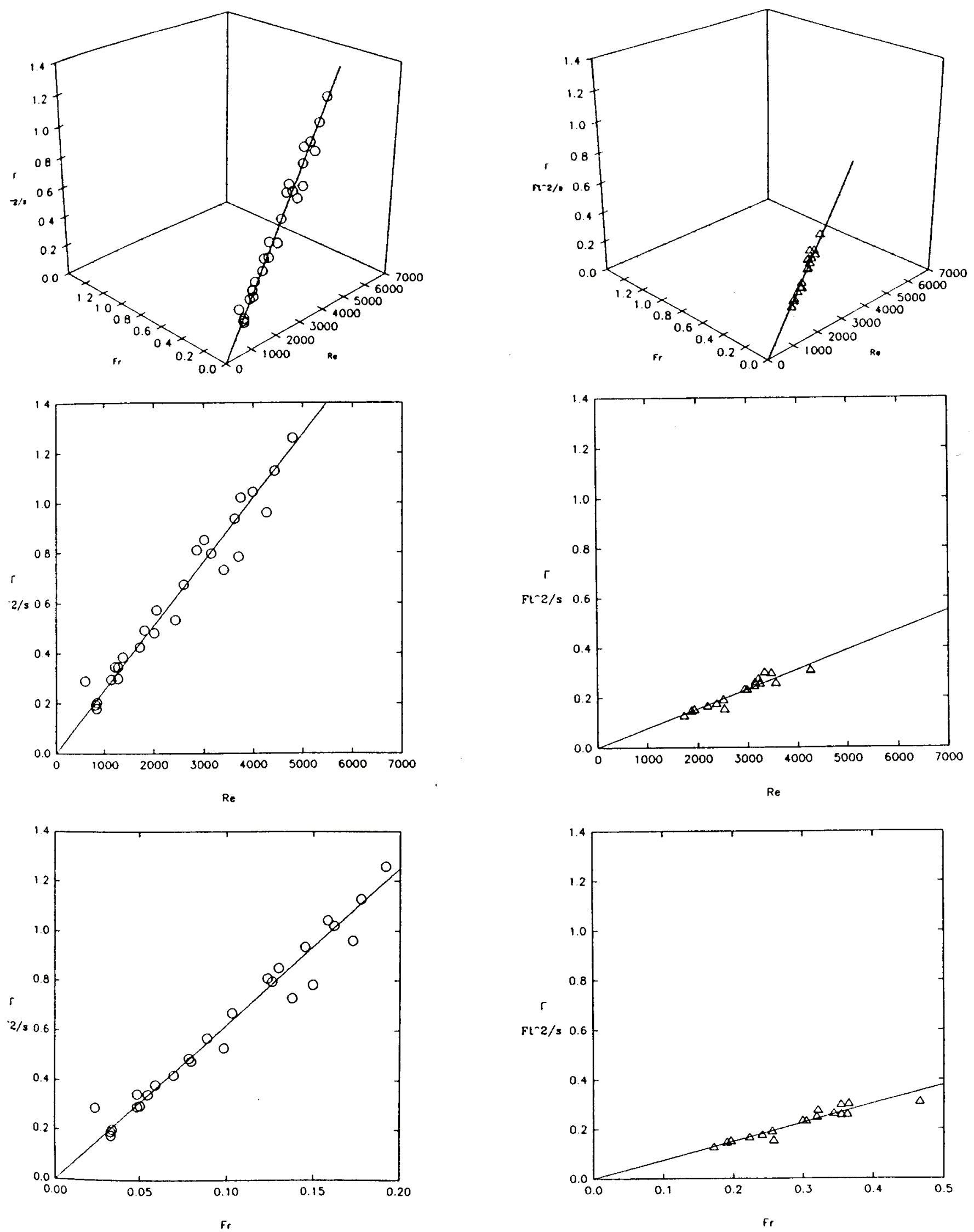

Figure 7. Circulation vs Reynolds and Froude numbers for $\mathrm{d}=1.588 \mathrm{~cm}$

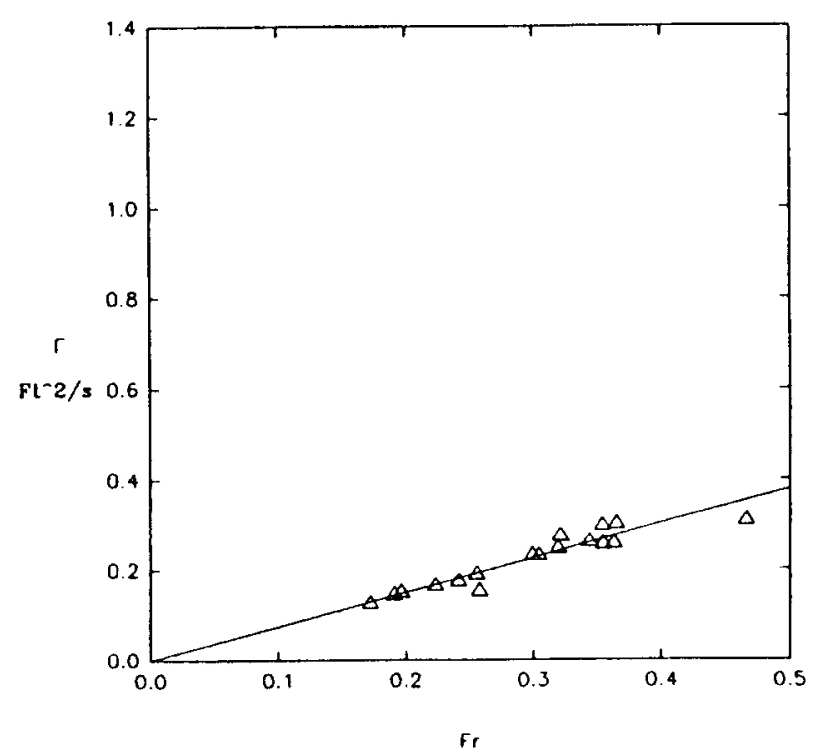

Figure 8. Circulation vs Reynolds and Froude numbers for $\mathrm{d}=0.635 \mathrm{~cm}$ 
Straight lines were fitted to the data in Figures 7 (a) and 8 (a); these lines were also projected into Figures 7 (b) \& (c) and 8 (b) \& (c). The slopes of these projections for the $\Gamma$ vs Reynolds and the $\Gamma$ vs Froude number graphs for all five drain diameters are plotted vs the ratio of the drain diameter to cylinder diameter in Figure 9. The equation of the lines fitted to the data in Figure 9 are:

$$
\frac{\partial\left(\frac{\Gamma}{v}\right)}{\partial(\operatorname{Re})}=229.1\left(\frac{d}{D}\right)
$$

and

$$
\frac{\partial\left(\frac{\Gamma}{v}\right)}{\partial(F r)}=554.2 \times 10^{5}\left(\frac{d}{D}\right)^{2}
$$
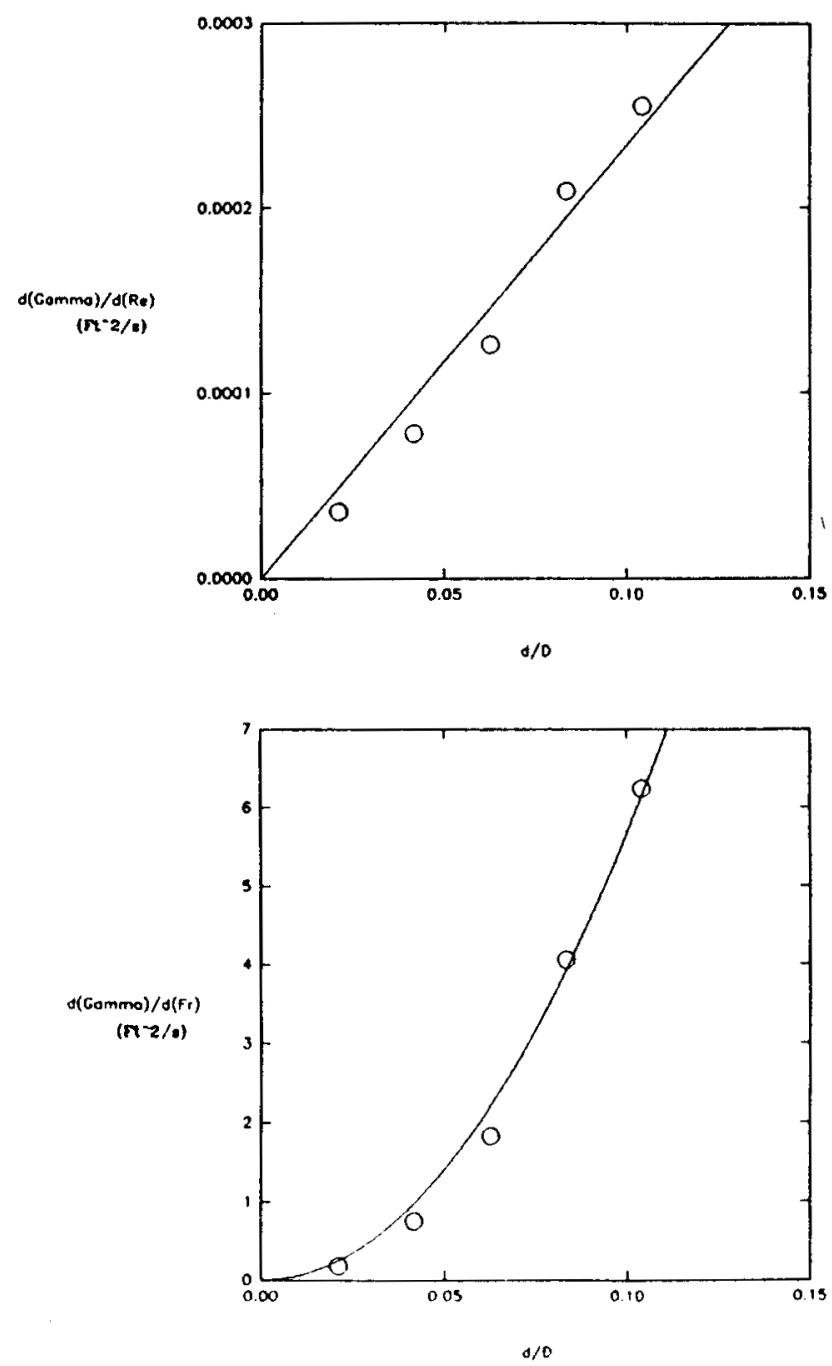

Figure 9. Dependence of the slopes of circulation vs Reynolds number and circulation vs Froude on $\mathrm{d} / \mathrm{D}$
Circulation thus appears to increase linearly with the drain velocity. From Figure 9 (a) and equation 10, it can be seen that the circulation is proportional to the ratio $d / D$ and the Reynolds number; the Reynolds number is based on the drain diameter, $d$, and therefore circulation should be proportional to the ratio (d/D) $)^{2}$ and the outlet velocity, $V$. This is verified by the results in Figure $9(\mathrm{~b})$ and equation 11.

The air core depth $h$ provides a qualitative measure of vortex strength; a smaller air core implies a weaker vortex while a larger air core implies a stronger vortex. There were some difficulties in measuring $h$. For small drain diameters or large flow rates, the tip of the vortex was not steady and oscillated about a mean value; for larger drain diameters or lower flow rates this was not a problem. After the flow rate was set, the air core took several minutes to fully develop. As the air core approached its maximum depth at large velocities, it "choked" the flow, the flow rate decreased slightly, and the size of the air core decreased. As the air core decreased, the flow increased, the vortex filaments stretched, and the air core increased in size and the process repeated.

Figures 10 (a) and 11 (a) show the dimensionless parameter h/H plotted against Reynolds and Froude numbers. Figures 10 (b) and 11 (b) show the projection of the experimental data on the Reynolds- $\mathrm{h} / \mathrm{H}$ plane. Figures 10 (c) and 11 (c) show the projection of the data on the Froude- $h / H$ plane. From the graphs it can be seen that the depth of the air core, h, increases with both the Reynolds and Froude numbers. The experimental data points exhibit a general " $S$ " shape. 

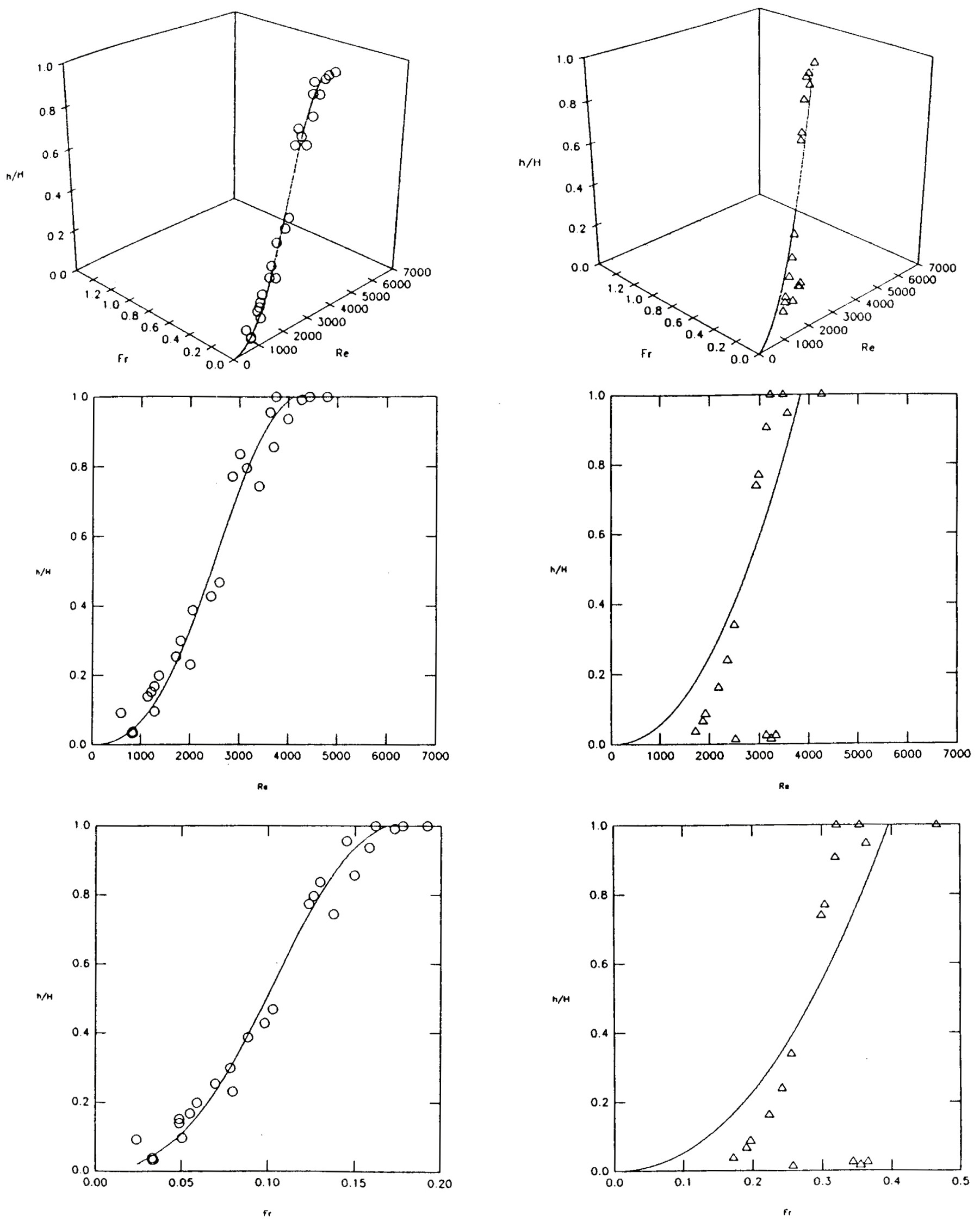

Figure 10. $h / H$ vs Reynolds and Froude numbers for $\mathrm{d}=1.588 \mathrm{~cm}$

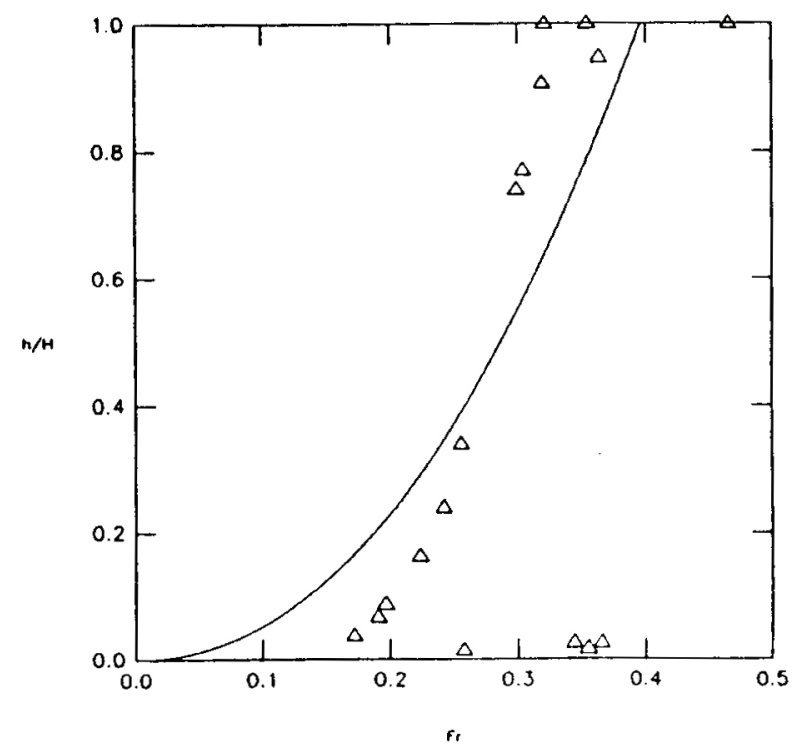

Figure 11. $\mathrm{h} / \mathrm{H}$ vs Reynolds and Froude numbers for $\mathrm{d}=0.635 \mathrm{~cm}$ 
Since circulation can now be easily measured, a relationship between circulation and the ratio $h / H$ would allow the depth of the air core to be predicted. In Figure $12, \Gamma / \Gamma_{\max }$ is plotted against $\mathrm{h} / \mathrm{H}$ for all five outlet diameters. $\Gamma_{\max }$ is the maximum circulation measured for any specific drain diameter when $\mathrm{h} / \mathrm{H}=1$. $\Gamma_{\max }$ is different for each drain diameter. As can be seen from the figure, the curves for $1.588 \mathrm{~cm}, 1.27 \mathrm{~cm}$, and $0.953 \mathrm{~cm}$ drain diameters fall on top of each other; the curves for $0.635 \mathrm{~cm}$ and $0.318 \mathrm{~cm}$ drain diameters also fall on top of each other, but slightly above the first three curves. It is suspected that the shift in the latter data points is due to the Weber number effects. The $0.635 \mathrm{~cm}$ and $0.318 \mathrm{~cm}$ drain diameters formed very thin air cores. The radius of curvature at the tip of the air core is affected by surfactants on the free surface. These results indicate that $\Gamma / \Gamma_{\max }$ is similar to $\mathrm{h} / \mathrm{H}$ in a global sense.

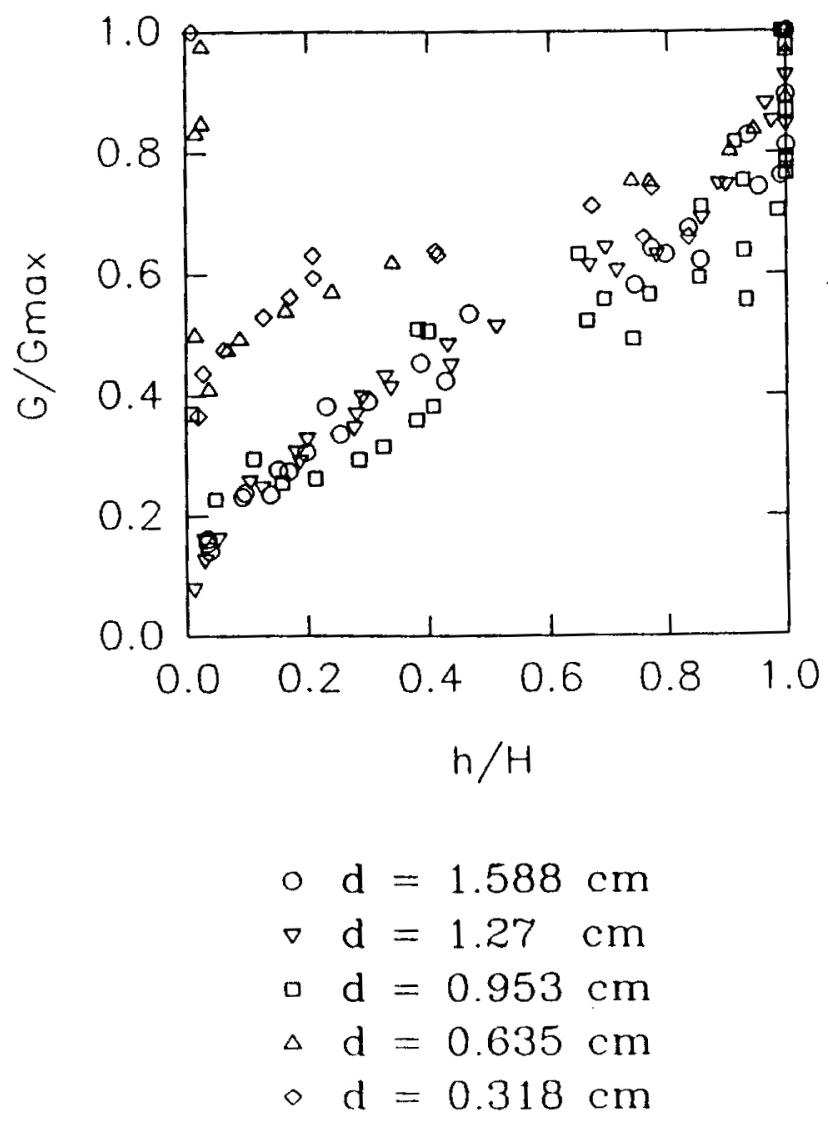

Figure 12. $\Gamma / \Gamma_{\max }$ vs $h / H$ for all five drain diameters tested

\section{Conclusions}

The ultrasonic transit-time difference measurement technique can be used to directly and non-intrusively measure the circulation of free surface vortices on a closed triangular path. The circulation of a vortex was found to increase linearly as the Reynolds and Froude numbers increased. The slopes of circulation versus Reynolds and Froude number curves increased as the drain diameter increased. The slope of circulation against Reynolds number increased linearly with $d / D$; whereas the slope of circulation against Froude number increased as (d/D) $)^{2}$. The ratio $\mathrm{h} / \mathrm{H}$ also increases as the Reynolds and Froude numbers increase and these curves are " $\mathrm{S}$ " shaped. Dependence of circulation and $\mathrm{h} / \mathrm{H}$ on the Weber number and the tank aspect ratio H/D were not studied. Weber number effects on circulation is expected to be small.

Further studies should investigate conditions where Reynolds and Froude numbers can be varied independently while holding the other parameters constant. This is difficult since Reynolds number variations have to be accommodated by viscosity changes while Froude number changes should be performed by changing $\mathrm{H}$. Moreover, larger Reynolds numbers have to be reached to asses the importance of viscous effects in industrial applications.

\section{Acknowledgements}

We would like to thank Lawrence C. Lynnworth of the Panametrics, Inc. for his assistance and the loan of special equipment, such as the PT 868 Portable Liquid Flowmeter. We would also like to thank S. Jacobson, J. True, and N. Talcherkar for their assistance during experimentation at the Panametrics facilities in Waltham, MA.

\section{References}

'Gluck, D. F.; Gille, J. P.; Simkin, D. J.; Zukoski, E. E., "Distortion of the Liquid Surface During Tank Discharge Under Low G Conditions," Chemical Engineeing Progress Symposium Series, Vol. 62, No. 61, 1966, pp. 150-157.

${ }^{2}$ Escudier, M., "Confined Vorticies in Flow Machinery," Annual Review of Fluid Mechanics, Vol. 19, 1987, pp. 27-52.

${ }^{3}$ Ramamurthi, K; Tharakan, T., "Shaped Discharge for Draining Ports," Journal of Spacecraft and Rockets, Vol. 30, Number 6, November-December 1993, pp. 786-788. 
"Anwar, H. O., "Prevention of Vortices at Intakes," Water Power, October, 1968, pp. 393-401.

'Motycka, D.; Muller, G.; Walter, W., "An Analytical and Experimental Study of Inlet Ground Vortices," AlAA Paper 73-1313, 1973.

${ }^{6}$ Brand, R.; Lubin, B., "Critical Height for Vortex Formation in a Liquid Draining from a Rotating Tank," Journal of Fluid Engineering, June 1977, pp. 1-36.

'Cuomo, A.; Farell, C., "Introduction to the Study of Vortex Dynamics and Similitude in Free Surface Flows," Technical Report, Centro Tecnologico de Hidraulica, Departamento de Aguas e Energia Electria, Sao Paulo, Brazil, 1981, pp. 1-82.

${ }^{8}$ Daggett, L. L.; Keulegan, G. H., "Similitude in FreeSurface Vortex Formations," Journal of the Hydraulics Division, ASCE, Vol. 100, No. HY 11, 1974, pp. 15651581 .

${ }^{9} \mathrm{Chang}, \mathrm{E}$. , "Review of the Literature on Drain Vortices in Cylindrical Tanks," BHRA Fluid Engineering, March 1976, pp. 1-31.

${ }^{10}$ Durgin, W. W.; Hecker, G. E., "The Modeling of Vortices at Intake Structures," The Joint Symposium of Design and Operation of Fluid Machinery, Fort Collins, Colorado, June 12-14, 1978, pp. 1-10.

"Lynnworth, L. C., "Ultrasonic Flowmeter Cell Designs for Liquids," Ultrasonics Symposium, Milwaukee, Wisconsin, November 11-14, 1974, pp. 1-4.

${ }^{12}$ Lowell, F., "The Design of Open Channel Acoustic Flowmeters, Part 1," Water Power and Dam Construction, April, 1978, pp. 49-55.

${ }^{13}$ Lowell, F., "Acoustic Flowmeters for Pipeline Flowrate," Water Power \& Dam Construction, June 1979 , pp. 39-46.

"Lowell, F., "The Design of Open Channel Acoustic Flowmeters for Specified Accuracy: Sources of Error and Calibration Test Results," Proceedings of the Symposium on Flow in Open Channels and Closed Conduits, Gaithersburg, Maryland, February 23-25, 1977, pp. 243-266.
15 Lowell, Jr., F., "Acoustic Flowmeters for Open Channels: Designing to Meet a Specified Accuracy," Flow Measurment of Fluids, Edited by $H$. $H$. Dijstelbergen and E. A. Spencer, North-Holland Publishing Company, 1978, pp. 371-378.

${ }^{16}$ Smith, Jr., R. H., "The Direct Measurement of Circulation in Free Surface Vortices," MS Thesis, Worcester Polytechnic Institute, Worcester, Massachusetts, April, 1994. 